\title{
Role of Alfuzosin in Ureteral Stent Related Urinary Symptoms Score: A Comparative Study
}

\author{
Shrestha $\mathrm{S}^{*}$, Adhikari $\mathrm{DB}^{1}$, Gurung $\mathrm{A}^{1}$, Pradhan $\mathrm{S}^{1}$, Gurung $\mathrm{NV}^{1}$, Acharya $\mathrm{A}^{1}$, Shrestha $\mathrm{D}^{1}$, Poudel $\mathrm{SR}^{1}$, Chapagain $\mathrm{A}^{1}$, \\ Shrestha $D^{1}$, Lamichhane $P^{2}$ \\ 'Department of Surgery, Western Regional Hospital, Pokhara, Nepal \\ ${ }^{2}$ Department of Microbiology, Faculty of Medicine, Siriraj Hospital, Mahidol University, Bangkok, Thailand
}

\author{
Keywords \\ Alfuzosin, Alpha blocker, Ureteral stent, \\ Urinary symptoms.

\section{Corresponding author} \\ *Dr. Santosh Shrestha \\ Consultant surgeon \\ Department of Surgery, \\ Western Regional Hospital \\ Pokhara, Nepal \\ E-mail: santos.stha@gmail.com
}

\begin{abstract}
Background: Ureteral stent placement is a routine urological procedure. However, patients inserted with ureteral stent exhibited increased urinary symptoms that compromise patients' quality of life.

Objective: To assess the efficacy of alpha blockers (Alfuzosin) in the management of ureteral stent related urinary symptoms.

Methods: Total of 60 patients after ureteral stent insertion was randomly divided into two equal groups; 30 in alfuzosin group and the remaining 30 in control group. Urinary symptoms questionnaire was filled after two weeks and results were statistically analyzed.
\end{abstract}

Results: Urinary symptoms like urgency, frequency and flank pain were significantly less in the alfuzosin group when compared with control group.

Conclusion: Alpha blocker (Alfuzosin) was found to be effective in reducing ureteral stent related urinary symptoms.

\section{INTRODUCTION}

Ureteral stent was first introduced by Zimskind in $1967^{1}$. With the development of double J (DJ) stent and pigtail stent by Finney ${ }^{2}$ and Hepperlen ${ }^{3}$ in 1978, placement of ureteral stent emerged as one of the most common procedures in urology. However, its placement results in different symptoms resulting in poor quality of patients' life. DJ stent is associated with different ureteral stent related symptoms like frequency, urgency, flank pain, suprapubic pain and hematuria ${ }^{4,5}$. The incidence varies from $19-76 \%{ }^{6-8}$.

The exact mechanism of DJ stent related symptoms have yet to be elucidated. It has been postulated that frequency is due to mechanical stimulation from bladder coil while urgency is due to direct presence of stent ${ }^{9}$. Ureteral stent cause a rise in intrapelvic pressure that might give rise to flank pain during vesicle filling or voiding ${ }^{10,11}$. Suprapubic pain was postulated to be due to local irritation of trigonal mucosa by the stent ${ }^{12}$. Hematuria results from insertion of the stent itself ${ }^{12}$.

Various approaches were taken to reduce ureteral stent related urinary symptoms in patients. Size, length and position of the stent play vital role in minimizing stent related urinary symptoms. Generally, alpha blockers ( $\alpha$-blockers) were used and found to be effective in reducing urinary symptoms in patients after ureteral stent insertion. However, the efficacy of alfuzosin, an 
alpha blocker in the management of ureteral stent related urinary symptoms in our setting needs to be determined.

\section{METHODS}

\section{Study subjects}

A randomized, prospective, comparative and experimental clinical trial was conducted between March 1, 2015 to November 30, 2016 at Western Regional Hospital, Pokhara, Kaski, Nepal. All the patients who underwent open surgery for renal stones, pyeloplasty, ureteric stones and after URSL with DJ stent placement were included in the study; while all the patients of pediatric age group were excluded. A total of 60 patients were divided into 2 equal groups; control group, and alfuzosin group those receiving alfuzosin $10 \mathrm{mg}$ OD. Antibiotics were given to both groups. Patients in alfuzosin group received alfuzosin (10 mg OD) on second day of surgery and continued for two weeks. All the patients were called on $14^{\text {th }}$ day of surgery and were asked to fill up a questionnaire addressing ureteral stent related urinary symptoms like frequency, urgency, flank pain, suprapubic pain and hematuria. The study protocol was evaluated and approved by the Department of Surgery, Western Regional Hospital, Pokhara, Kaski, Nepal.

\section{Statistical analysis}

Data were plotted using Graph Pad Prism version 5.0 (Graph Pad software Inc., La Jolla, CA). Chi-square test was used to analyze the data set. A P-value $<0.05$ was considered statistically significant.

\section{RESULTS}

Total 60 patients were recruited in this study (30 in each group; alfuzosin group and control group). Ureteral stent related symptoms are summarized in Table 1 . Symptoms were compared between the alfuzosin and control groups for all 60 study subjects. Total five urinary symptoms were measured. It was found that the symptoms were reduced in alfuzosin group compared with control groups. In particular, use of alfuzosin significantly reduced three of the five urinary symptoms; specifically frequency (58.35\%, $P=0.044)$, urgency $(69.99 \%, P=0.028)$ and flank pain $(87.51 \%, P=0.011)$ (Table 1$)$. This suggested that alfuzosin helps in reducing urinary stent related symptoms in patients.
Table 1: Urinary stent related symptom scores in patients

\begin{tabular}{lcccc}
\hline \multicolumn{1}{c}{ Urinary } \\
symptoms & $\begin{array}{c}\text { Alfuzosin } \\
\text { group } \\
(\mathbf{n = 3 0 )}\end{array}$ & $\begin{array}{c}\text { Control } \\
\text { group } \\
(\mathbf{n}=30)\end{array}$ & $\begin{array}{c}\text { Percent } \\
\text { reduction } \\
\text { (Alfuzosin } \\
\text { vs control) }\end{array}$ & $\begin{array}{c}\boldsymbol{P} \\
\text { value }\end{array}$ \\
\hline Frequency & $5(16.66 \%)$ & $12(40 \%)$ & 58.35 & $0.044^{*}$ \\
Urgency & $3(10 \%)$ & $10(33.33 \%)$ & 69.99 & $0.028^{*}$ \\
Flank Pain & $1(3.33 \%)$ & $8(26.66 \%)$ & 87.51 & $0.011^{*}$ \\
$\begin{array}{l}\text { Suprapubic } \\
\text { pain }\end{array}$ & $4(13.33 \%)$ & $6(20 \%)$ & 33.35 & 0.488 \\
Hematuria & 0 & $2(6.66 \%)$ & 100 & 0.158 \\
\hline
\end{tabular}

*Statistically significant using chi-Square test $(P<0.05)$

\section{DISCUSSION}

In management of ureteral stent related urinary symptoms $\alpha$-blockers were used and found to be effective. Herein, we assessed the efficacy of $\alpha$-blocker, alfuzosin in our setting. To judge the therapeutic benefit of alfuzosin, patients (alfuzosin group or control group) inserted with DJ stent were asked to fill a questionnaire addressing five ureteral stent related urinary symptoms.

It has been shown that DJ stent insertion is associated with increased urinary symptoms. This study showed that DJ stent insertion has increased the frequency, flank pain and suprapubic pain to $40,33.33$ and $20 \%$, respectively. This is in agreement with previous meta-analysis wherein frequency, urgency and suprapubic pain has been shown to be $50-60 \%, 57-60 \%$ and $30 \%$, respectively ${ }^{7,13}$.

Moreover, this study showed that urgency and hematuria has been experienced by 33 and $7 \%$ of cases, respectively. This is lower than what had been observed previously, i.e. $57-60 \%$ and $25 \%$ of cases experienced urgency and hematuria, respectively ${ }^{6,7}$. The discrepancy might be due to limited number of cases in the present study (60) compared with $>1,500$ cases in those study ${ }^{6,7}$. Also, the questionnaires were filled at two weeks in the present study compared with six weeks in the other study ${ }^{6}$.

Although the use of $\alpha$-blocker significantly reduced most of the ureteral stent related urinary symptoms compared with control groups, robust study that recruits a large number of cases needs to be studied for a better conclusion. Moreover, other factors like size, length and position of the stent that were shown to affect patients' quality of life after ureteral stent insertion should be considered along with use of $\alpha$-blockers in the management of ureteral 
stent related urinary symptoms.

\section{Conflict of interest}

No conflict of interest to declare

\section{REFERENCES}

1. Zimskind PD, Fetter TR, Wilkerson JL. Clinical use of long-term indwelling silicone rubber ureteral splints inserted cystoscopically. J Urol. 1967; 97(5): 840-4.

2. Finney RP. Experience with new double J ureteral catheter stent. J Urol. 1978; 120(6): 678-81.

3. Hepperlen TK, Mardis HK. Pigtail stent termed means of lessening ureteral surgery. Trends Clin Urol. 1978: 1-405.

4. Zhou L, Cai X, Li H, Wang KJ. Effects of alpha-Blockers, antimuscarinics, or combination therapy in relieving ureteral stent-related symptoms: A meta-analysis. $J$ Endourol. 2015; 29(6): 650-6.

5. Haleblian G, Kijvikai K, de la Rosette J, Preminger G. Ureteral stenting and urinary stone management: A systematic review. J Urol. 2008; 179(2): 424-30.

6. He F, Man LB, Li GZ, Liu N. Efficacy of alpha-blocker in improving ureteral stent-related symptoms: A metaanalysis of both direct and indirect comparison. Drug Des Devel Ther. 2016; 10: 1783-93.
7. Hao P, Li W, Song C, Yan J, Song B, Li L. Clinical evaluation of double-pigtail stent in patients with upper urinary tract diseases: Report of 2685 cases. $J$ Endourol. 2008; 22(1): 65-70.

8. Joshi HB, Stainthorpe A, MacDonagh RP, Keeley FX, Jr., Timoney AG, Barry MJ. Indwelling ureteral stents: Evaluation of symptoms, quality of life and utility. $J$ Urol. 2003; 169(3): 1065-9; discussion 1069.

9. Joshi HB, Okeke A, Newns N, Keeley FX, Jr., Timoney AG. Characterization of urinary symptoms in patients with ureteral stents. Urology. 2002; 59(4): 511-6.

10. Mosli HA, Farsi HM, al-Zimaity MF, Saleh TR, alZamzami MM. Vesicoureteral reflux in patients with double pigtail stents. J Urol. 1991; 146(4): 966-9.

11. Ramsay JW, Payne SR, Gosling PT, Whitfield HN, Wickham JE, Levison DA. The effects of double J stenting on unobstructed ureters. An experimental and clinical study. Br J Urol. 1985; 57(6): 630-4.

12. Miyaoka R, Monga M. Ureteral stent discomfort: Etiology and management. Indian J Urol. 2009; 25(4): 455-60.

13. Zhang P, Hu WL, Cheng B, Cheng L, Zeng YJ, Wang G. alpha1-blockers for the reduction of ureteric stentrelated symptoms: A systematic review and metaanalysis. Exp Ther Med. 2016; 11(2): 660-68. 